Robert Kreher*, Thomas Groscheck, Kristjan Qarri, Bernhard Preim, Alexander Schmeisser, Thomas Rauwolf, Rüdiger Christian Braun-Dullaeus and Sandy Engelhardt

\title{
A novel calibration phantom for combining echocardiography with electromagnetic tracking
}

https://doi.org/10.1515/cdbme-2020-0003

\begin{abstract}
Ultrasound compounding techniques offer the possibility to enlarge the otherwise limited field of view of ultrasound. However, existing works mainly rely on larger ultrasound sensors. In this work, we attach electromagnetic (EM) tracking sensors to small tubular echo probes, namely an intracardiac echocardiographic (ICE) probe and a transesophageal echocardiographic (TEE) transducer. The EM tracking allows, when synchronized to the ultrasound, localization of the probes in either $5 \mathrm{DOF}$ (Degrees of Freedom) or 6 DOF without line-of-sight requirement. For computation of the references between the two systems, we developed a novel customized 3D-printable phantom, which is especially convenient for tubular probes that acquire images laterally. Calibration with the phantom and 3D volume reconstruction was conducted in the Plus Toolkit. The volume reconstructor uses the captured position and orientation information to fuse 2D ultrasound slices into a compounded volume. Mean calibration error is below $2.5 \mathrm{~mm}$ for ICE and TEE. An accuracy evaluation of the 3D reconstruction using an object of known geometry revealed that tracking with 5 DOF provides unsatisfactory results, while the combination of 6 DOF and TEE achieved a mean absolute difference of $3.08 \mathrm{~mm}$. Our calibration phantom fCal-Echo1.0 is openly available at http://perk-software.cs.queensu.ca/plus/doc/ nightly/modelcatalog/.
\end{abstract}

\footnotetext{
*Corresponding author: Robert Kreher, Research Campus STIMULATE, Magdeburg, Germany; and Faculty of Computer Science, Otto-vonGuericke University, Magdeburg, Germany,

E-mail: robert.kreher@ovgu.de

Thomas Groscheck, Research Campus STIMULATE, Magdeburg, Germany; Faculty of Computer Science, Otto-von-Guericke University, Magdeburg, Germany

Kristjan Qarri and Bernhard Preim, Faculty of Computer Science, Ottovon-Guericke University, Magdeburg, Germany

Alexander Schmeisser, Thomas Rauwolf and Rüdiger Christian Braun-Dullaeus, Research Campus STIMULATE, Magdeburg, Germany; Department of Cardiology, Magdeburg University Hospital, Magdeburg, Germany

Sandy Engelhardt, Research Campus STIMULATE, Magdeburg, Germany; Heidelberg University Hospital, Heidelberg, Germany
}

Keywords: calibration phantomICE; ultrasound; plustoolkit; volume reconstruction

\section{Introduction}

Echocardiography is a fast, flexible, and inexpensive medical imaging modality. It is an invaluable tool in preoperative diagnosis, intraoperative guidance, and postoperative monitoring of patients with cardiovascular diseases. Different examinations range from transesophageal echocardiography (TEE), over transthoracic echocardiography (TTE) to intracardiac echocardiography (ICE), which all require different probes. In case of TEE, a small probe is inserted in the esophagus and 2D/3D images are captured in different positions. In TTE, the ultrasound (US) transducer is placed on the chest to allow for a noninvasive examination of cardiac structures. In case of ICE, a small ultrasound probe is integrated at the tip of a catheter, which allows imaging from inside the heart.

However, despite being an acquisition method that also allows for high frame rates, echocardiography is inherently limited by a small field of view. Hence, image compounding methods are an attractive solution to increase the field-of-view. This is particularly interesting for ICE approaches, which are often hampered by a loss of orientation during maneuvering of the catheter.

Existing ultrasound compounding methods, which were mainly developed for other application areas, are either image-based or guided by an additional position sensor, such as an electromagnetic (EM) tracking sensor [1]. EM has the advantage to track inside the body of the patient without line of sight to the field generator. In our scenario, the position sensor must be located directly at the tip of the small probes and is therefore very close to the piezoelectric elements, which might especially impact tracking accuracy.

Existing work [1, 2] showed that EM tracking accuracy must be evaluated for each individual application and setup. Therefore, the contribution of our work is threefold:

(1) We integrate a novel openly available calibration phantom into an existing open-source calibration procedure to link and synchronize EM tracking with ICE and TEE probes. 
(2) We assess the accuracy of EM tracking in the presence of echocardiography probes.

(3) Using the volume reconstruction plugin of the Plus toolkit, we perform a 3D-volume reconstruction of the captured 2D-slices on an object of known geometry and report the reconstruction error.

\section{Methods}

We use NDI's Aurora system (Northern Digital Inc., Waterloo, Ontario, Canada) Version 3 with a Planar Field Generator because it offers good portability, can be easily combined with the mentioned open source software, and the tracking sensors can be fixed to the probes. Our NDI Aurora system was delivered with a factory calibration error of $2.3 \mathrm{~mm}$ at the largest possible distance between field generator and 6 DOF sensor.

\subsection{Novel calibration phantom}

To link image and position data, a calibration process must be carried out. The Plus toolkit (v.2.8.0.2019) already provides a widely used calibration method, that is based on detecting wires in sectional US views of a phantom [3]. Plus itself offers three models (fCal-2.0, fCal2.1, fCal-3.1) for download on its website ${ }^{1}$. Two of them are applicable for ultrasound probes with low penetration depth and the other is suitable for high penetration depth. However, none of them is optimized for working with tubular probes or NDI Aurora.

Therefore, a calibration phantom is needed which must fulfill the following properties: First, a reference senor must be fixable to the phantom that has a standardized distance to the origin of the phantom, eliminating the need to measure it manually (R1). Second, a dense pattern of thin wires must be tightened accurately inside the phantom. They are visible in the small field of view of the US image and therefore serve as basis for calibration (R2). Third, a special cavity needs to be spared from the phantom's wall to facilitate image acquisition with tubular probes that acquire images laterally (R3).

We addressed these requirements and some more details are provided in the following. In comparison to the existing calibration phantoms, the number of holes for the wires was increased and their sizes were reduced to a scale that is still 3D-printable. Circular holes were replaced by triangles, which allow for a more accurate positioning of the wires, since they are pushed into the same corners of the opposing triangles when placing tension on the wire and therefore lie on a straight line. Unlike the existing phantoms with only 14 points on three sides, the new phantom has 28 possible registration points on five sides to increase accuracy and accessibility (R2). To improve integration with NDI Aurora, two possible positions for the reference sensor were incorporated (R1). This eliminates the need to manually measure the displacement between the reference sensor to the origin of the phantom (Figure 1). The phantom is freely available to download. ${ }^{1}$ It is recommended to print it with a resin (SLA/DLP) 3D-printer as this offers high resolution and therefore higher accuracy.

1 Plus toolkit website with models: http://perk-software.cs.queensu. ca/plus/doc/nightly/modelcatalog/.
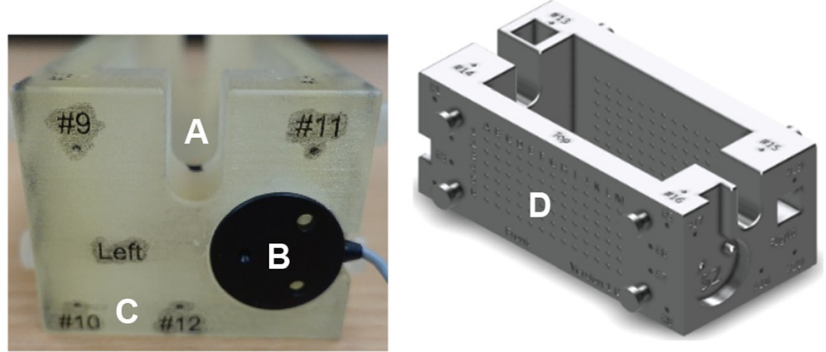

Figure 1: (A) Channel for catheter, (B) Reference sensor embedded in the phantom, (C) Registration points, (D) Attachment grid for wires.

\subsection{Tracked echocardiography probes}

Two tracking setups were created. First, a Z6M TEE probe (Siemens Healthineers GmbH, Erlangen, Germany) was equipped with a 6 DOF sensor (Figure 2). Second, two smaller 5 DOF sensors were attached with super glue on opposite sides to the head of a Siemens AcuNav ICE catheter (Figure 3). For the latter case, the system was configured such that the tracking information is interpolated between the two sensors.

\section{Experiments and results}

Several experiments were conducted. The first experiment addresses the interference between ICE/TEE probe with EM tracking and was conducted in a laboratory. The second experiment is conducted to assess the reconstruction error using image compounding algorithms.
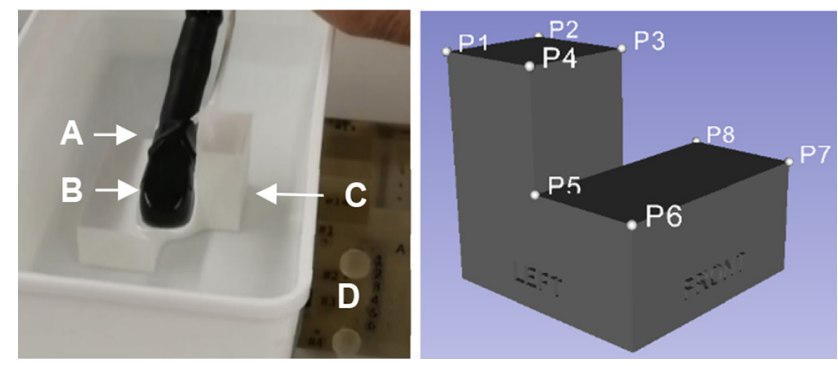

Figure 2: Left: Image acquisition setup. (A) 6 DOF sensor, (B) head of the TEE probe, (C) test phantom, (D) calibration phantom attached to the tank. Right: Cubic test phantom with eight landmark points.

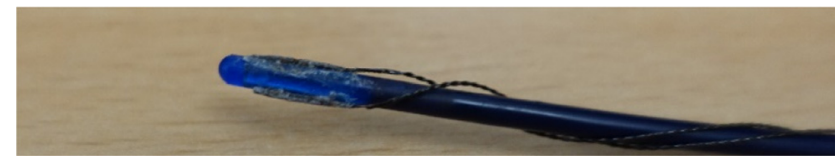

Figure 3: Head of the ICE catheter with two 5 DOF sensors. 

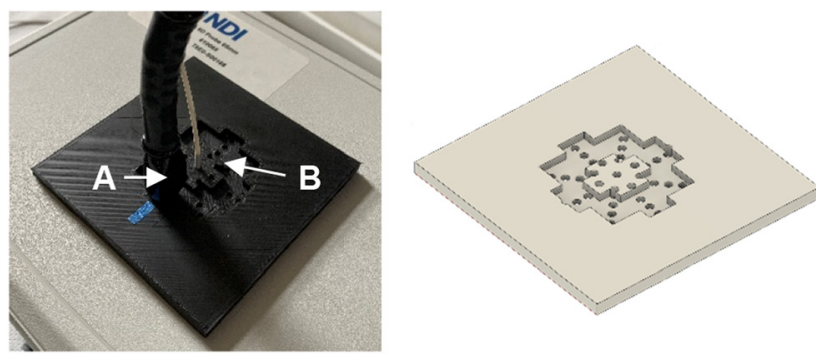

Figure 4: Left: Accuracy acquisition setup. (A) head of the TEE probe, (B) 6 DOF sensor. Right: Plate rendering.

\subsection{EM-tracking accuracy in the presence of echo probes}

A special board was created (Figure 4) to measure potential influence of the echo probe to the EM tracking accuracy. EM sensor was fixed to the centre of the board and the field generator was placed below. Different relative positions of the echo probes were tested for the 5 and 6 DOF sensors. The TEE probe was placed in a circle at eight positions at a distance of $1 \mathrm{~cm}$ around the respective sensor. The orientation of the probe was kept the same. For the ICE catheter the same was done with 32 positions. The experiments were carried out with the ultrasound probe in an active and inactive state, to determine the effect on the EM tracking. The settings on the ultrasound machine were selected so that the highest possible ultrasound power was emitted.

The results show, that there is only a minimal difference of $0.045 \pm 0.024 \mathrm{~mm}$ for the 6 DOF Sensor and $0.041 \pm 0.111 \mathrm{~mm}$ for the $5 \mathrm{DOF}$ Sensor in accuracy, when the TEE probe is active. With the ICE catheter, the error for the 6 DOF Senor was $0.045 \pm 0.031 \mathrm{~mm}$ and for the 5 DOF $0.018 \pm 0.026 \mathrm{~mm}$. This shows that the accuracy of EM tracking is not noticeably affected by the ultrasound probes.

\subsection{Calibration accuracy}

The second experiment was carried out on the table of a cone-beam CT angiography laboratory site, which resembles the future application environment. The field generator was fixed to the bottom side of the head's position of the table. A tank filled with degassed water and the calibration phantom was fixed to the top side where the heart should be located.

Subsequently, the calibration procedure in the Plus toolkit was repeated 10 times for each probe. The attempt with the minimal error of $2.03 \mathrm{~mm}$ for the ICE probe (mean $2.21 \pm 0.21 \mathrm{~mm}$ ) and $1.78 \mathrm{~mm}$ for the TEE probe (mean
$2.40 \pm 0.31 \mathrm{~mm})$ was chosen for all subsequent reconstructions. ${ }^{2}$

\subsection{D-compounding accuracy}

A test object was designed and 3D-printed as a reference with known geometry that should be reconstructed by 3D-echo compounding. It is designed as a simple composite of four equal cubes each with an edge length of $25 \mathrm{~mm}$. The simple shape allows for determination of the 3D-corner points on the virtual, real and imaged object (Figure 2). As ground truth, we have chosen the distances between eight corner points (Figure 2). We additionally measured these eight points with an Aurora 4 DOF straight probe. This procedure was repeated five times.

To verify the accuracy of the print, the dimensions were checked with a digital caliper gauge with a precision of $0.1 \mathrm{~mm}$. The results are reported in Table 1.

The test phantom was scanned once each with the TEE probe and the ICE catheter by manually moving the probe over the phantom and the lateral acquisitions were collected. The settings of the Siemens SC2000 ultrasound machine were not changed during the acquisition process. The test setup was not moved between passes.

Due to inaccessibility of raw data, images had to be collected via a frame grabber. Unnecessary image information was removed with the aid of a mask such that only the area of the US cone is further analysed. Subsequently, a 3D volume was created with the help of the Plus toolkit (VolumeReconstructor plugin) using the 2D images with

Table 1: Difference between virtual ground truth (GT) edge lengths and the real object (calliper measurement and stylus measurement) and the US compounded image (TEE and ICE). Mean absolute difference (MAD) is reported in the last row.

\begin{tabular}{lrrrrr}
\hline Edge & $\begin{array}{r}\text { Virtual } \\
\text { GT edge } \\
\text { length }\end{array}$ & $\begin{array}{r}\text { Calliper } \\
\text { stylus }\end{array}$ & $\begin{array}{r}\text { Aurora 6 } \\
\text { DOF } \\
\text { sty }\end{array}$ & $\begin{array}{r}\text { 6 DOF TEE } \\
\text { compounding }\end{array}$ & $\begin{array}{r}\text { 5 DOF ICE } \\
\text { com-pounding }\end{array}$ \\
\hline P1-P2 & 25.00 & 0.00 & -0.11 & +1.67 & -0.69 \\
P1-P4 & $\sim$ & 0.10 & -0.20 & +3.78 & -4.20 \\
P3-P2 & $\sim$ & 0.10 & 0.20 & +5.20 & -3.88 \\
P3-P4 & $\sim$ & 0.00 & -0.34 & -0.15 & -1.63 \\
P4-P5 & $\sim$ & 0.10 & +0.48 & -3.42 & - \\
P5-P6 & 50.00 & 0.20 & +0.27 & +1.45 & - \\
P5-P8 & 25.00 & 0.00 & -0.50 & +3.73 & - \\
P7-P6 & 25.00 & 0.00 & +0.29 & +6.18 & - \\
P7-P8 & 50.00 & 0.20 & +0.20 & +2.11 & +3.49 \\
MAD & - & 0.08 & 0.29 & 3.08 & 3.07 \\
\hline
\end{tabular}

2 The specifications of the Plus toolkit recommend an error of less than $3 \mathrm{~mm}$. 


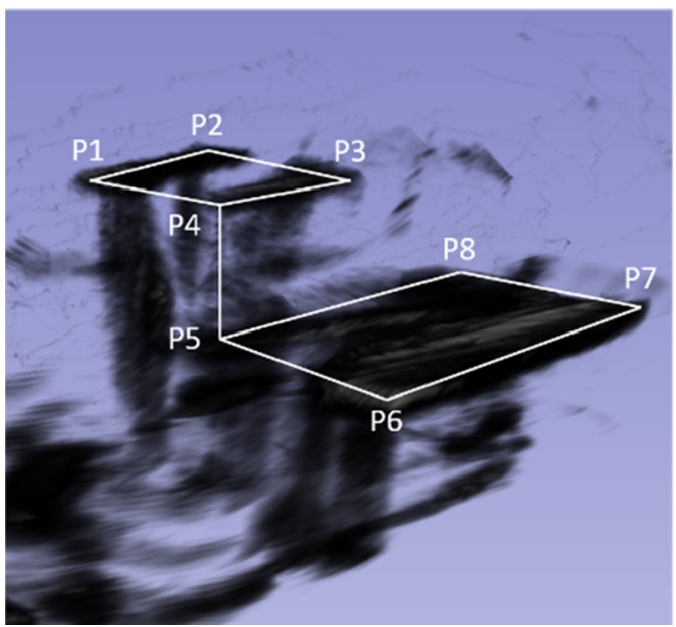

Figure 5: 3D-reconstructed test object captured with a tracked TEE probe. Compounding is according to the transformation information from the 6 DOF EM-Sensor.

the corresponding position and orientation determined by EM-tracking and the calibration file.

The corner points of the test object were manually determined in the reconstructed US volume using 3D Slicer [4]. This process was repeated five times and the average is used to calculate the distances between the different points (Figure 5 and Table 1).

The reported results indicate that EM tracking alone is able to determine the edge lengths in sub-millimetre range with a 6 DOF stylus. The accuracy of the actual point measurements are accurate to the hundredth. The accuracy of the stylus measurement is $0.066 \pm 0.037 \mathrm{~mm}$.

The volume reconstruction with the ICE catheter and the $2 \times 5$ DOF sensors was insufficient such that only six points could be identified and five distances could be calculated.

\section{Discussion}

The customization of the calibration phantom on the NDI Aurora system and catheter has greatly simplified the calibration workflow by eliminating the need for adjustments between the reference sensor and phantom. Mean calibration error was below $2.5 \mathrm{~mm}$ in both settings and interference between EM and US is marginal.

Volume compounding was possible in the 6 DOF setting with the TEE probe. Difficulties arose in the other setting, leading to a situation where only six of eight corner points of the test object could not be identified. However, the overall quality of the compounding needs to be improved and further investigations on the influence of the manual swipe direction and position need to be conducted. We assume that the existing compounding method in the Plus toolkit was probably developed for probes that deliver a higher quality image and capture a larger field of view.

Research funding: This work is funded by the European Regional Development Fund under the operation number 'ZS/2016/04/78123' as part of the initiative "SachsenAnhalt WISSENSCHAFT Schwerpunkte".

Author contributions: All the authors have accepted responsibility for the entire content of this submitted manuscript and approved submission.

Conflict of interest: Authors state no conflict of interest.

\section{References}

1. Franz AM, Haidegger T, Birkfellner W, Cleary K, Peters TM, MaierHein L. Electromagnetic tracking in MedicineA review of technology, validation, and applications. In: IEEE Trans Med Imaging; 2014, Vol. 33, pp. 1702-25.

2. Hastenteufel M, Vetter M, Meinzer H, Wolf I. Effect of 3D ultrasound probes on the accuracy of electromagnetic tracking systems. Ultrasound Med Biol 2006;32:1359-68.

3. Lasso A, Heffter T, Rankin A, Pinter C, Ungi T, Fichtinger G. PLUS: open-source toolkit for ultrasound-guided intervention systems. In: IEEE Trans Biomed Eng; 2014, Vol. 61, pp. 2527-37.

4. Fedorov A, Beichel R, Kalpathy-Cramer J, Finet J, Fillion-Robin J, Pujol S, et al.. 3D slicer as an image computing platform for the quantitative imaging network. Magn Reson Imaging 2012;30: 1323-41. 